\title{
Linking Social Media Posts to News with Siamese Transformers
}

\author{
Jacob Danovitch \\ Institute for Data Science \\ Carleton University \\ Ottawa, CA \\ jacob.danovitch@carleton.ca
}

\begin{abstract}
Many computational social science projects examine online discourse surrounding a specific trending topic. These works often involve the acquisition of large-scale corpora relevant to the event in question to analyze aspects of the response to the event. Keyword searches present a precision-recall trade-off and crowd-sourced annotations, while effective, are costly. This work aims to enable automatic and accurate ad-hoc retrieval of comments discussing a trending topic from a large corpus, using only a handful of seed news articles.
\end{abstract}

\section{Introduction}

Many computational social science projects examine online discourse surrounding a specific trending topic, such as political events, natural disasters, or sporting matches. These works often involve the acquisition of large-scale corpora relevant to the event in question to analyze aspects of the response to the event, often with a focus toward social media. However, existing methods for the acquisition of these events come with trade-offs.

Commonly, keyword searches are used (23), which present a choice between high precision (using narrow keywords and getting fewer but more accurate results) and recall (using broad keywords and getting many irrelevant results). One common method of keyword searching, specific to Twitter, is the use of hashtags. However, hashtag-only searches can lead to low recall (17). Crowd sourcing is an effective way to filter corpora (18), though it can be expensive both monetarily as well as in terms of time spent. This work proposes a method for automatic and accurate ad-hoc retrieval of comments discussing a trending topic from a large corpus using only a handful of seed news articles. Using a Siamese architecture and triplet loss, we jointly embed news articles and social media posts with the objective of minimizing the distance between the embeddings of each article and its relevant posts. This allows the automatic filtering of corpora by selecting comments most similar to news articles describing the event or topic of interest. The asymmetric lengths of comments and news articles pose a challenge for Siamese architectures. We propose a novel solution using a sparse attention mechanism, which allows the network to attend only to the most relevant parts of the input, easing the asymmetry in length. We make our code publicly available ${ }^{1}$

\section{Related Work}

The specific task of matching articles and social media comments (used interchangeably with posts) has received limited attention, especially in recent years. Some early works focused on social content alignment, the task of aligning comments to specific sentences within articles. Latent Dirichlet

\footnotetext{
1 https://github.com/jacobdanovitch/jdnlp
} 
Allocation $(29,12)$ and several extensions, such as the Document-Comment Topic Model (14; 13) and Specific-Correspondance LDA (5), were used to provide interpretable alignments of comments to the most relevant segments of a news article. However, binary classification with feature engineering was seen to outperform these methods (30).

Similar methods have been employed for the task of matching tweets to news articles. (11) proposes a graphical weighted matrix factorization approach while also contributing a large-scale dataset similar to ours. Recent work using this dataset proposes an interactive attention mechanism to match tweet-article pairs (34). These works find the most relevant news article for a particular tweet, rather than vice versa. The intent of our work is more similar to (6), which identifies the most relevant tweet for a given news article using binary classifiers as well as semantic similarity.

Little has been done to apply more recent advancements in deep learning to this task. This is likely due to the large computational overhead in training over corpora of news articles, which consist of thousands of tokens each, as well as the inherent theoretical challenges these methods face in processing long documents. These challenges have inspired creative solutions, such as segmenting long documents into individual sentences, hierarchically processing each sentence, and performing an aggregation step (2). Alternatively, (19) presents an approach using Graph Convolution Networks on concept-interaction graphs to match pairs of news articles. While this is an effective method, the approach is restricted to pairwise classification, which creates a combinatorial explosion of pairs. Our work uses a Siamese architecture to perform retrieval using only cosine similarity.

\section{Methodology}

\subsection{Task definition}

We use a large corpus of tweets which share links to news articles published by CNN and the New York Times (11). The corpus contains 34,888 tweets which link to 12,704 unique articles. While the goal of the original study is to find the most relevant news article for a given tweet, we are interested in the reverse; finding the most relevant tweets for a given news article. We say that for each tweet-article pair $(T, A), T$ is relevant to $A$, and any other tweet $T^{\prime}$ not paired with $A$ is irrelevant.

\subsection{Notation}

We refer to vectors in bold $(\mathbf{x})$, tensors in capitalized bold $(\mathbf{X})$, and scalars in standard font $(x, X)$. We define an encoder as a deep neural network which maps a sequence of $S$ word embeddings $\left[e_{1}, e_{2}, \ldots, e_{S}\right]$ to either an output sequence of $D$-dimensional hidden states $\boldsymbol{H}=$ $\left[\boldsymbol{h}_{1}, \boldsymbol{h}_{2}, \ldots, \boldsymbol{h}_{S}\right]$ (in the sequence-to-sequence case) or a single $D$-dimensional representation $\boldsymbol{H}$ (in the sequence-to-vector case).

\subsection{Objectives}

We wish to use a Siamese network to jointly learn fixed-length embeddings for each $(T, A)$ pair, minimizing the distance between them using triplet loss. Projecting $A$ and $T$ into a common latent space allows us to compare the similarity of documents from vastly different domains (short, informal tweets versus long, formal news articles). However, doing so requires an encoder capable of solving several challenges pertaining to document length.

Noise. News articles can be in the order of thousands of tokens, and they often discuss multiple different topics (13). The asymmetric length of tweets relative to the articles suggests that tweets rarely discuss the entirety of the article's content, making much of the article irrelevant and perhaps even adding additional noise. As such, it would be useful for an encoder to be able to filter out this noise and focus on only the most important input tokens.

Efficiency. Using large batch sizes creates more informative triplets (33), which is constrained by the already-high memory usage of processing thousands of tokens at once. These concerns are accentuated by the computational demands of the attention mechanism. While attention is more effective than other methods for modelling long sequences, its $\mathcal{O}\left(n^{2}\right)$ complexity (31) will exhaust 
reasonable resources with such long documents. As such, we require an encoder capable of efficiently processing long documents without sacrificing quality in the process.

\subsection{Blocking out the noise}

We begin by confronting the theoretical challenges of modelling long documents, such as the ability to capture the long-range dependencies and focusing only on crucially important tokens. Attention-based architectures have shown to outperform alternative candidates for these challenges (28). However, certain models such as BERT (7) can be computationally intensive and impose maximum token lengths, which prevents their use. An additional problem for attention-based models introduced by such long documents is that they may struggle to identify vital portions of the text. For example, consider the attention mechanism and its multi-headed extension (31):

$$
\begin{aligned}
\operatorname{Attention}(\mathbf{Q}, \mathbf{K}, \mathbf{V}) & =\operatorname{softmax}(f(\mathbf{Q}, \mathbf{K})) \mathbf{V} \\
\boldsymbol{a}_{\boldsymbol{i}} & =\operatorname{Attention}\left(\boldsymbol{Q} \boldsymbol{W}_{\boldsymbol{Q}_{i}}, \boldsymbol{K} \boldsymbol{W}_{\boldsymbol{K}_{i}}, \boldsymbol{V} \boldsymbol{W}_{\boldsymbol{V}_{i}}\right) \\
\text { MultiHeadAttention }(\mathbf{Q}, \mathbf{K}, \mathbf{V}) & =\left[\boldsymbol{a}_{\mathbf{1}} ; \boldsymbol{a}_{\mathbf{2}} ; \ldots \boldsymbol{a}_{\boldsymbol{h}}\right]
\end{aligned}
$$

Where the parameters $\boldsymbol{W}_{\boldsymbol{Q}_{i}}, \boldsymbol{W}_{\boldsymbol{K}_{i}}, \boldsymbol{W}_{\boldsymbol{V}_{i}} \in \mathbb{R}^{D}$ are trainable and $f$ is a function which computes the

similarity between each entry in $\boldsymbol{Q}$ and $\boldsymbol{K}$. One such function is $\cos (\mathbf{Q}, \mathbf{K})=\frac{\mathbf{Q K}^{\mathbf{T}}}{\|\mathbf{Q}\| \cdot \mathbf{K}^{\mathbf{T}} \|}$, which is known as cosine attention (9). Crucial to the attention operation is the softmax function:

$$
\sigma(\boldsymbol{z})_{i}=\frac{e^{z_{i}}}{\sum_{j=1}^{S} e^{z_{j}}}
$$

This produces an $S$-length vector with a sum of 1 , where each $z_{i}$ is a weight for each token in the input document. The operation $\operatorname{softmax}(\cdot) \boldsymbol{V}$ is equivalent to taking a weighted average of each token embedding in $\boldsymbol{V}$. Note that softmax has full support (all $\sigma(z)_{i}>0$ ), which means that even unimportant portions of the input document will still receive non-zero weight (4). As we noted earlier, our documents are very long and many of the words could be unimportant to the output, which makes it highly difficult for softmax to filter them out without assigning any weights of precisely 0 . In fact, we observe a negative correlation between $S$ and $\mathbb{E}\left[\sigma(\boldsymbol{z})_{i}\right]$ - that is, as documents grow longer, the expected weight of a given token decreases. We provide a brief proof in appendix A

This is problematic, as it will be difficult for the most important tokens to stand out in such long sequences like news articles. As the value of each attention weight decreases, the distribution becomes more uniform, and the operation becomes equivalent to simply taking the mean over all $\boldsymbol{h}_{\boldsymbol{i}} \in \boldsymbol{H}$, many of which should have been filtered out. To counter this problem, we look toward the growing family of sparse activation functions. Figure 1 demonstrates a comparison of self attention using softmax versus one such sparse function, known as sparsemax (22). While both activations identify important tokens in a sequence of 5 , softmax approaches uniformity for the sequence of length 25 while sparsemax still identifies important tokens.

In particular, we replace softmax with the $\boldsymbol{\alpha}$ - entmax activation function, a controllably sparse alternative (27), and we use a unique trainable parameter $\boldsymbol{\alpha}_{h}$ for each attention head $h$. This is known as adaptively sparse multi-head attention, which addresses our first objective by allowing each attention head to learn an optimal level of sparsity (4). This allows each head to learn which segments of the input sequence to filter, reducing the noise introduced by long input documents.

\subsection{Improving efficiency}

Next, we address our second objective, which requests an encoder capable of efficiently processing long documents without sacrificing quality in the process. Irrespective of the activation function used, the $\mathcal{O}\left(n^{2}\right)$ complexity of a standard Transformer layer is prohibitively expensive for documents thousands of tokens long. As such, we require a way to model long-range dependencies more efficiently than a standard Transformer. For this, we use the Star Transformer, a simple and efficient extension of the standard Transformer capable of learning many of the same semantic relationships and long-range dependencies (10). 


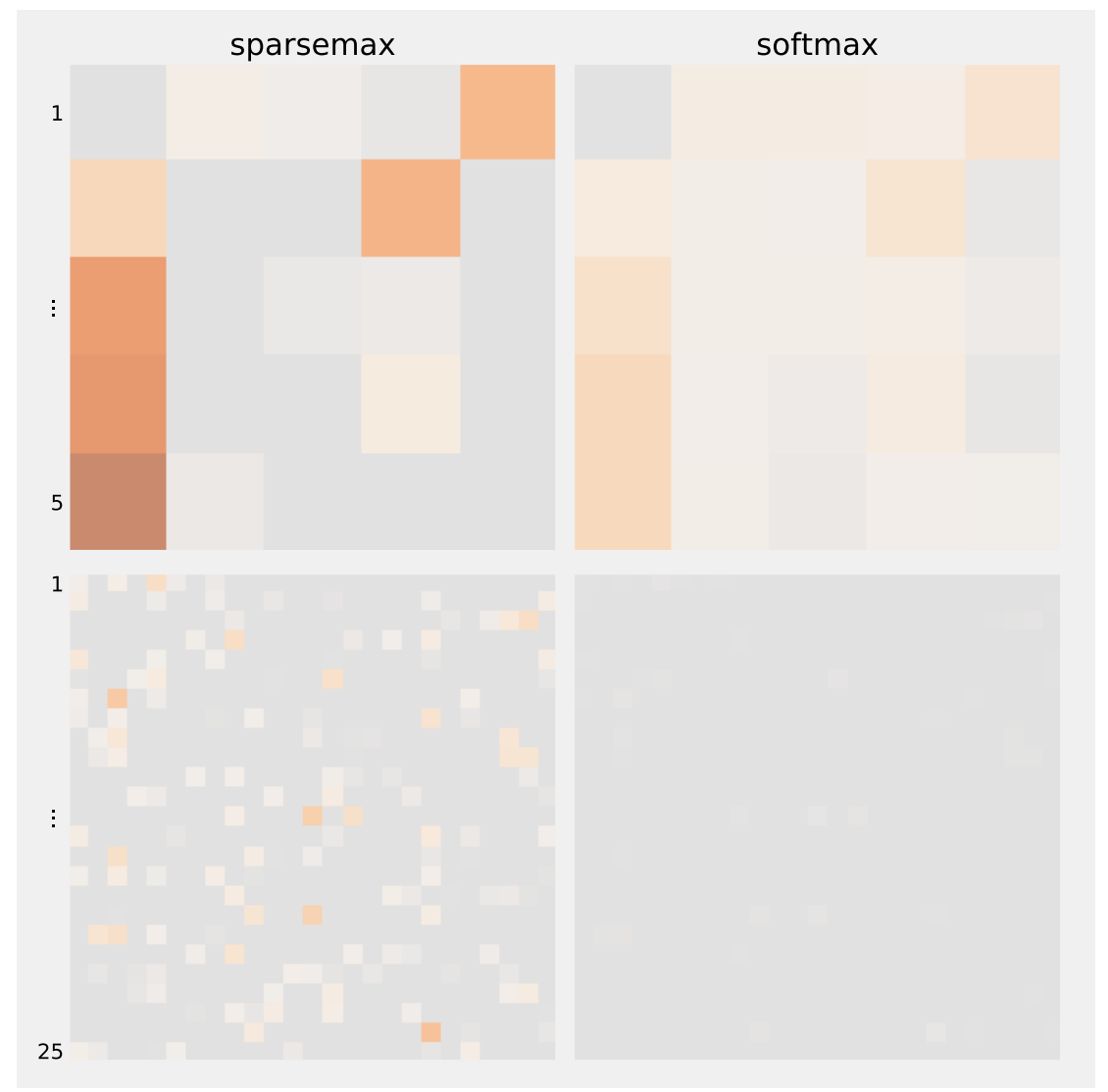

Figure 1: Self-attention applied to sequences of 5 and 25 random embeddings using softmax and sparsemax. As document length increases, softmax produces nearly uniform weights approaching 0 , making self-attention equivalent to taking the mean over all embeddings.

The Star Transformer is able to reduce the quadratic time complexity to linear time $(\mathcal{O}(n))$ by using a message-passing mechanism along a sliding window of embeddings. Given a sequence of input embeddings $\left[\mathbf{e}_{1}, \mathbf{e}_{2}, \ldots, \mathbf{e}_{n}\right]$, we produce an output sequence of embeddings $\left[\mathbf{h}_{\mathbf{1}}, \mathbf{h}_{\mathbf{2}}, \ldots, \mathbf{h}_{\boldsymbol{n}}\right]$. Initially, each $\mathbf{h}_{i}=\mathbf{e}_{i}$, and a message-passing relay node $\mathbf{s}$ is initialized as the mean of all $\mathbf{e}_{i}$. For each embedding $\mathbf{e}_{i}$, its immediate $c$ neighbors and the relay node $\mathbf{s}$ are used to form a new representation $\mathbf{h}_{i}$. This message-passing mechanism enables the learning of long-range dependencies in linear time, as each attention operation only considers the $c$ immediate neighbors of each token (as well as the relay node).

$$
\begin{aligned}
\mathbf{C}_{i} & :=\left[\mathbf{h}_{i-c} ; \ldots \mathbf{h}_{i} ; \ldots \mathbf{h}_{i+c} ; \mathbf{s}\right] \\
\mathbf{h}_{i} & :=\operatorname{MultiHeadAttention}\left(\mathbf{C}_{i}, \mathbf{h}_{i}, \mathbf{h}_{i}\right)
\end{aligned}
$$

After all $i$ operations, we then update the relay node as $\mathbf{s}:=\operatorname{MultiHeadAttention}(\mathbf{H}, \mathbf{s}, \mathbf{s})$ where $\mathbf{H}=\left[\mathbf{h}_{\mathbf{1}} ; \mathbf{h}_{\mathbf{2}} ; \ldots \mathbf{h}_{\boldsymbol{n}}\right]$. The authors find that performing $T$ iterations of these operations enables re-reading to more effectively capture long-range dependencies, though additional iterations only marginally improved performance in our experiments. To encode the output sequence $\mathbf{H}$ to a fixedlength vector, we take the maximum over each embedding dimension and average it with the final state of the relay node, computed as $\frac{\max (\mathbf{H})+\mathbf{s}}{2}$.

\subsection{Siamese Architecture}

Finally, we use our altered Star Transformer to jointly embed each tweet-article pair, represented as a tuple of word embedding sequences $\left(\left[\boldsymbol{t}_{1}, \boldsymbol{t}_{2}, \ldots, \boldsymbol{t}_{m}\right],\left[\boldsymbol{a}_{1}, \boldsymbol{a}_{2}, \ldots, \boldsymbol{a}_{n}\right]\right)$. Each are independently 


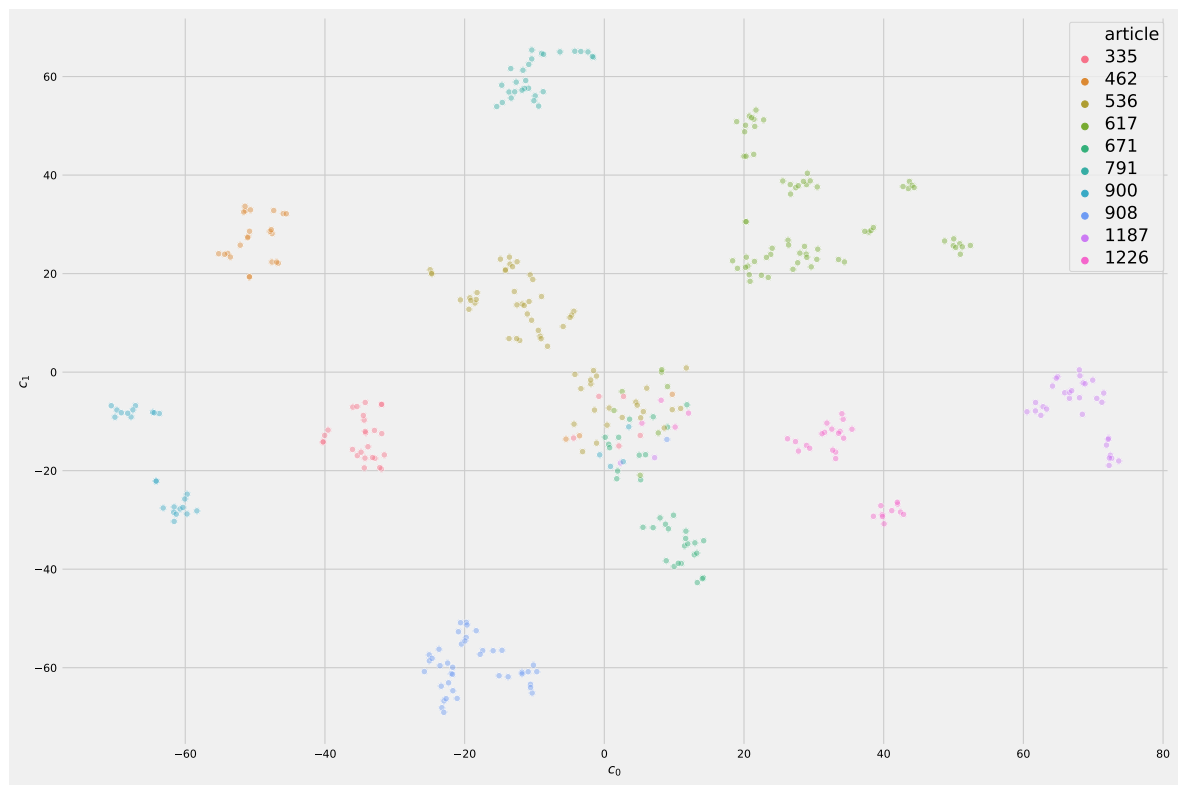

Figure 2: t-SNE projection of tweets about the most shared news articles.

passed through the encoder, producing fixed-length vectors $f_{t}$ and $f_{a}$. These embeddings are directly passed to the objective function, triplet loss (1), formulated as:

$$
L=\max \left(0,\left\|\boldsymbol{f}_{\boldsymbol{a}}-\boldsymbol{f}_{\boldsymbol{t}}\right\|^{2}-\left\|\boldsymbol{f}_{\boldsymbol{a}}-\boldsymbol{f}_{\boldsymbol{t}^{\prime}}\right\|^{2}+\alpha\right)
$$

Where $f_{t^{\prime}}$ is an irrelevant tweet mined using the multi-similarity method (32), and $\alpha$ is the margin. This minimizes the distance between the embeddings of articles and their relevant tweet(s). Figure 2 shows the t-Stochastic Neighbor Embedding (t-SNE) (20) projection of the learned embeddings for a sample of tweets which reference the top-10 most shared articles in the dataset. The result is that tweets referring to the same articles are tightly clustered in latent space. We call our resulting model the Adaptive Siamese Transformer (AST).

\section{Experimental Results}

\subsection{Evaluation Criteria}

The goal of our work is to enable the automatic filtering of a corpus of tweets using news articles as seeds. We assume that it is cheap to collect tweets, but expensive to annotate them, and that an ideal model identifies relevant tweets while returning as close to 0 false positives as possible.

Precision, a common metric for many information retrieval tasks, reflects our wish to minimize the number of false positives. A model that retrieves exclusively relevant documents will have a precision of $100 \%$. However, this could be achieved by simply returning a very small amount of relevant documents. We ensure that a reasonable number of documents are returned by using $r$-Precision as our evaluation metric, which computes the precision after $r$ documents are retrieved. We also report several other metrics for additional comparison.

\subsection{Inference Process}

For evaluation, we hold out approximately 3300 samples as our test set. The model returns an embedding for each news article $f_{a}$ and tweet $f_{t}$. The relevance score of a given $f_{a_{i}}$ to a given $f_{t_{j}}$ is defined as their cosine similarity. 


\subsection{Training Configuration}

We train Siamese networks on a large dataset of tweet-article pairs (11) using three additional encoders as baselines, including Convolutional Neural Networks (CNN) (16), Gated Recurrent Units (GRU) (3), and Bidirectional GRUs (Bi-GRU) as benchmarks. Each network uses 300-dimensional pre-trained GloVe embeddings (26). Triplet mining was performed using the pytorch_metric_learning library (24), and experiments were conducted with the AllenNLP library (8) using four NVIDIA V100 GPUs on Google Cloud Platform. All configuration details are available on GitHub.

\subsection{Results}

\begin{tabular}{|l|r|r|r|r|}
\hline & CNN & GRU & Bi-GRU & AST \\
\hline 50 & $\mathbf{1 0 0 . 0}$ & 98.00 & 98.00 & $\mathbf{1 0 0 . 0}$ \\
\hline 100 & $\mathbf{9 9 . 0 0}$ & 96.00 & $\mathbf{9 9 . 0 0}$ & 96.00 \\
\hline 200 & $\mathbf{9 7 . 5 0}$ & 92.00 & 96.50 & 94.50 \\
\hline 500 & 90.40 & 88.00 & $\mathbf{9 2 . 6 0}$ & 92.40 \\
\hline 1000 & $\mathbf{8 4 . 8 0}$ & 79.80 & 83.70 & $\mathbf{8 4 . 8 0}$ \\
\hline 2000 & 68.65 & 62.25 & 67.25 & $\mathbf{7 0 . 0 0}$ \\
\hline 3000 & 55.53 & 49.93 & 54.27 & $\mathbf{5 7 . 9 0}$ \\
\hline
\end{tabular}

Table 1: $r$-Precision for each model at various thresholds.

Evaluation \#1. We report the $r$-Precision for each baseline encoder, which is the precision after having retrieved $r$ documents. For each $r$, we retrieve the $r$ highest scoring tweet-article pairs by cosine similarity, and compute the precision. This captures both the quality and quantity of documents retrieved by each model. All models decrease in precision as they retrieve more documents, as is expected (21). The GRU-based framework quickly falters in performance, demonstrating the challenges recurrent models face with long sequences. Bi-directionality appears to help, but the most competitive baseline was clearly the CNN-based framework. We posit that CNNs' ability to identify the most important n-grams using global max pooling (15) provides the filtering ability we describe in our first objective. Our model performs the best at higher quantity thresholds, significantly out-performing the recurrent models. It is possible that the sliding-window approach of the Star Transformer could lead to similar behavior as CNNs, a possibility we leave to be examined in future work.

\begin{tabular}{|c|c|c|c|}
\hline & mAP & AUC-ROC & mRP \\
\hline GRU & 43.53 & 96.03 & 76.68 \\
\hline Bi-GRU & 47.58 & 96.05 & 80.29 \\
\hline CNN & 49.81 & 97.09 & 81.05 \\
\hline AST & $\mathbf{5 3 . 0 1}$ & $\mathbf{9 7 . 5 0}$ & $\mathbf{8 1 . 3 1}$ \\
\hline
\end{tabular}

Table 2: Comparison of mean Average Precision, area under the Receiver-Operating Curve, and mean $r$-Precision between all models.

Evaluation \#2. We also report mean $\mathbf{r}$-Precision $(\mathbf{m R P})$, Mean average precision $(\mathbf{m A P})$, and area under the Receiver-Operating Curve (AUC-ROC). $\mathbf{m R P}$ is simply the $r$-Precision over all thresholds in Table 1 mAP and AUC-ROC are calculated using the scikit-learn library (25). Our model outperforms the three baselines in each metric.

Learned sparsity. Following the training process, we examine the $\alpha$ values learned by each attention head of the Star Transformer. Values closer to 1 will lead to attention weights closer to those produced by softmax, while values closer to 2 will be similar to sparsemax, and a value of 1.5 is equal to 1.5 - entmax (4). The star attention heads are used to update the relay node, while the ring attention heads are used at each context window. Overall, it appears that the ring attention heads learn a diverse set of alphas, allowing for varying levels of sparsity in the representations of each context window. Heads 4 and 5 approach sparsemax, while heads 3 and 6 remain relatively close to softmax. The $\boldsymbol{\alpha}$ values for the star attention heads were more tightly bound, but heads 1 and 3 were still quite sparse, exceeding the sparsity of 1.5 - entmax. 


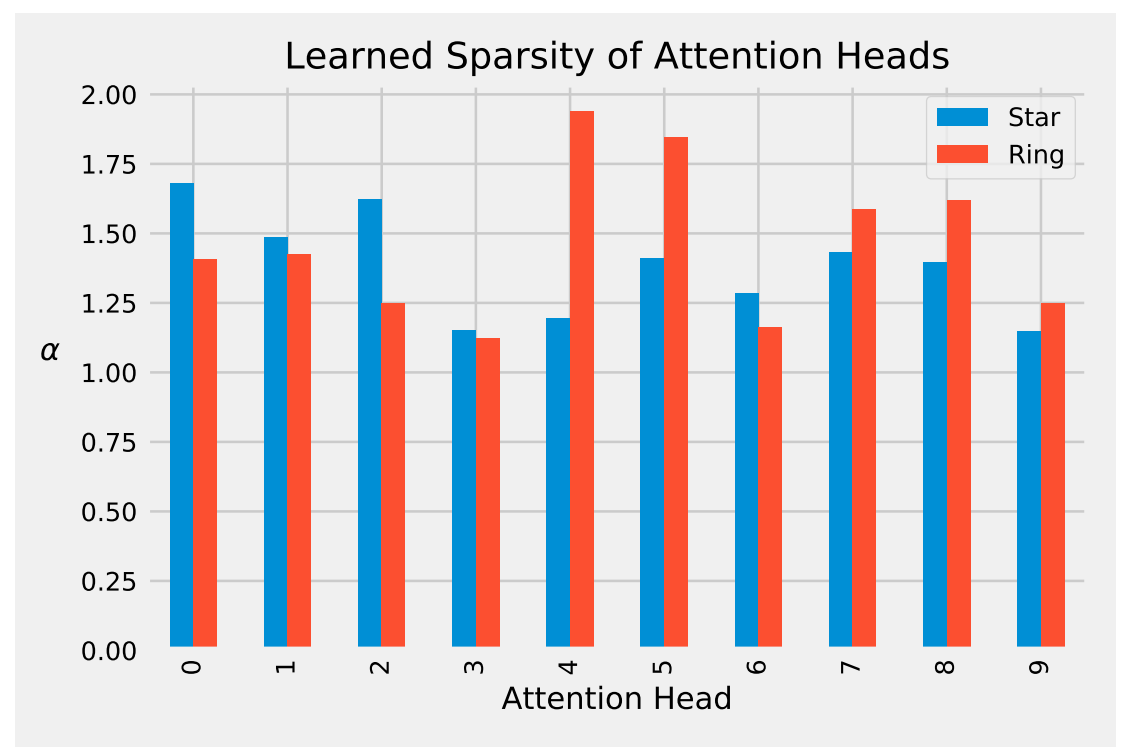

Figure 3: Learned $\boldsymbol{\alpha}$ for each attention head.

\section{Case Study}

Finally, we present a case study applying our model to a corpus of tweets about a terrorist attack which took place in Toronto, Canada in April 2018. We computed the cosine similarity of 100 manually annotated tweets' embeddings to that of a news article written shortly after the attack $\mathrm{K}^{2}$ As shown in Figure 4, with a retrieval threshold of $\cos \left(f_{a}, f_{t}\right) \geq 0.7$, our model was able to minimize the number of false negatives without greatly diminishing the quantity of tweets retrieved.

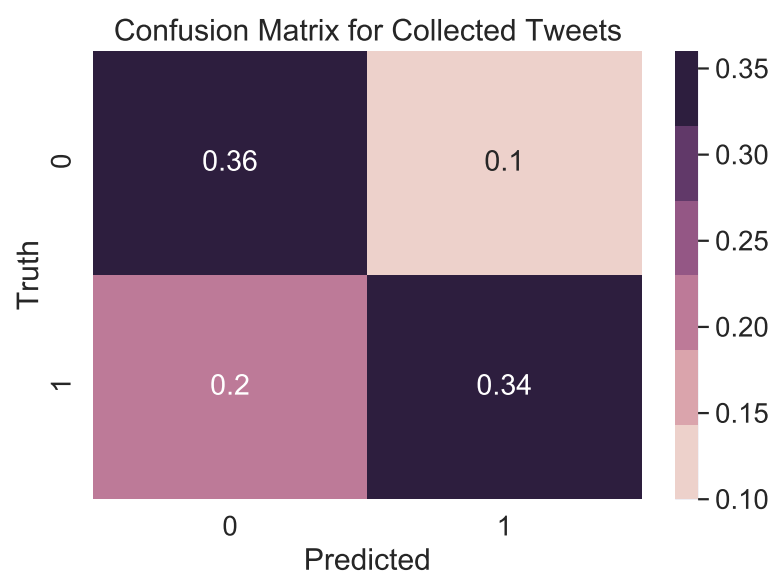

Figure 4: Of all tweets retrieved by our model, 36 were relevant while only 10 were irrelevant.

As seen in Table 3, sorting the tweets by cosine similarity showed results which may suggest that the model was able to learn which tweets are more relevant than others (as opposed to a binary notion of relevance), though we note these results are heavily anecdotal. We share these results to demonstrate potentially useful applications of our model. For example, a project which requires at least $N$ relevant tweets and precisely 0 irrelevant tweets necessarily requires human annotations. Our model could significantly decrease the financial cost of collecting annotations by presenting the tweets in order of estimated relevance (cosine similarity), minimizing the quantity of tweets that must be shown to annotators until $N$ relevant tweets are found.

2 https://globalnews.ca/news/4161785/pedestrians-hit-white-van-toronto/ 


\begin{tabular}{ll}
\hline tweet & score \\
\hline $\begin{array}{l}\text { Second, my thoughts and prayers go out to everyone in Toronto after the attack that } \\
\text { left } 10 \text { dead and } 16 \text { injured! A d also, thank you so much for a... }\end{array}$ & 0.9166 \\
What a devastating Tragedy! Savagery of mankind has no bound! Deadly Toronto van & 0.8996 \\
driver: What we know about Alek Minassian http://bit.ly/2vFJfDx & \\
Everyone at Community Safety Net would like to express their deepest sympathies to & 0.8841 \\
all those affected by yesterday's events in Toronto \#TorontoStr... & \\
What we know about Alek Minassian, the man charged in deadly Toronto van attack & 0.8838 \\
http://ow.ly/jZpd30jF9Jo & 0.8686 \\
$\begin{array}{l}\text { Yesterday was a deeply sad day for the city of \#Toronto and our hearts go out to the } \\
\text { victims, the families and anyone affected by the tragic incid... }\end{array}$ & $\vdots$ \\
$\begin{array}{l}\text { Incel or MGTOW Facebook groups banned me from their Facebook groups all because } \\
\text { they also hate not just women. }\end{array}$ & 0.4311 \\
$\begin{array}{l}\text { Une pense pour le tragique vnement Toronto } \\
\text { Stay strong Canada - we are with you }\end{array}$ & 0.3877 \\
$\begin{array}{l}\text { It is both wrong and unhelpful to cast the incel phenomenon as some kind of dark } \\
\text { internet cult. This is garden variety misogyny and rape culture w... }\end{array}$ & 0.3797 \\
$\begin{array}{l}\text { Reading that InCel "female ratings card" post going around and am increasingly beset } \\
\text { by a burning curiosity as to where lesbians fit into their co... }\end{array}$ & 0.3361 \\
\hline
\end{tabular}

Table 3: The most and least similar Tweets to a news article written after the attack.

\section{Discussion}

In conclusion, we present a novel approach to article-comment linking using a Siamese architecture and triplet loss. We encode pairs using Adaptive Star Transformers, an efficient Transformer using adaptively sparse attention to filter irrelevant information from the input sequences, which we show outperforms several other encoders in a Siamese framework. This model could allow other researchers to eliminate or vastly reduce the cost of filtering corpora.

\section{Acknowledgements}

Thank you to Kevin Musgrave for assistance using his pytorch_metric_learning library, and to Jorge Salazar for consultation on the proof regarding the softmax function and general proof-reading. 


\section{References}

[1] Gal Chechik, Varun Sharma, Uri Shalit, and Samy Bengio. Large scale online learning of image similarity through ranking. Journal of Machine Learning Research, 11(Mar):1109-1135, 2010.

[2] Muhao Chen, Changping Meng, Gang Huang, and Carlo Zaniolo. Neural article pair modeling for wikipedia sub-article matching. Lecture Notes in Computer Science, page 3-19, 2019.

[3] Kyunghyun Cho, Bart van Merrienboer, Caglar Gulcehre, Dzmitry Bahdanau, Fethi Bougares, Holger Schwenk, and Yoshua Bengio. Learning phrase representations using rnn encoder-decoder for statistical machine translation. Proceedings of the 2014 Conference on Empirical Methods in Natural Language Processing (EMNLP), 2014.

[4] Gonçalo M. Correia, Vlad Niculae, and André F. T. Martins. Adaptively sparse transformers. Proceedings of the 2019 Conference on Empirical Methods in Natural Language Processing and the 9th International Joint Conference on Natural Language Processing (EMNLP-IJCNLP), 2019.

[5] Mrinal Kanti Das, Trapit Bansal, and Chiranjib Bhattacharyya. Going beyond corr-lda for detecting specific comments on news \& blogs. In Proceedings of the 7th ACM International Conference on Web Search and Data Mining, WSDM '14, pages 483-492, New York, NY, USA, 2014. ACM.

[6] Orhan Demirsoz and Rifat Ozcan. Classification of news-related tweets. Journal of Information Science, 43(4):509-524, 2017.

[7] Jacob Devlin, Ming-Wei Chang, Kenton Lee, and Kristina Toutanova. Bert: Pre-training of deep bidirectional transformers for language understanding, 2018.

[8] Matt Gardner, Joel Grus, Mark Neumann, Oyvind Tafjord, Pradeep Dasigi, Nelson F. Liu, Matthew Peters, Michael Schmitz, and Luke S. Zettlemoyer. Allennlp: A deep semantic natural language processing platform. 2017.

[9] Alex Graves, Greg Wayne, and Ivo Danihelka. Neural turing machines, 2014.

[10] Qipeng Guo, Xipeng Qiu, Pengfei Liu, Yunfan Shao, Xiangyang Xue, and Zheng Zhang. Star-transformer, 2019.

[11] Weiwei Guo, Hao Li, Heng Ji, and Mona Diab. Linking tweets to news: A framework to enrich short text data in social media. In Proceedings of the 51st Annual Meeting of the Association for Computational Linguistics (Volume 1: Long Papers), pages 239-249, 2013.

[12] Tuan-Anh Hoang-Vu, Aline Bessa, Luciano Barbosa, and Juliana Freire. Bridging vocabularies to link tweets and news.

[13] Lei Hou, Juanzi Li, Xiao-Li Li, Jie Tang, and Xiaofei Guo. Learning to align comments to news topics. ACM Transactions on Information Systems (TOIS), 36(1):9, 2017.

[14] Lei Hou, Juanzi Li, Xiaoli li, Jiangfeng Qu, Xiaofei Guo, Ou Hui, and Jie Tang. What users care about: A framework for social content alignment. pages 1401-1407, 082013.

[15] Alon Jacovi, Oren Sar Shalom, and Yoav Goldberg. Understanding convolutional neural networks for text classification. In Proceedings of the 2018 EMNLP Workshop BlackboxNLP: Analyzing and Interpreting Neural Networks for NLP, pages 56-65, Brussels, Belgium, November 2018. Association for Computational Linguistics.

[16] Yoon Kim. Convolutional neural networks for sentence classification. Proceedings of the 2014 Conference on Empirical Methods in Natural Language Processing (EMNLP), 2014.

[17] Julie Letierce, Alexandre Passant, John Breslin, and Stefan Decker. Understanding how twitter is used to spread scientific messages. 2010.

[18] Xiaojie Lin. Linking news and tweets. 2016. 
[19] Bang Liu, Di Niu, Haojie Wei, Jinghong Lin, Yancheng He, Kunfeng Lai, and Yu Xu. Matching article pairs with graphical decomposition and convolutions, 2018.

[20] Laurens van der Maaten and Geoffrey Hinton. Visualizing data using t-sne. Journal of machine learning research, 9(Nov):2579-2605, 2008.

[21] Christopher D Manning, Christopher D Manning, and Hinrich Schütze. Foundations of statistical natural language processing. 1999.

[22] André F. T. Martins and Ramón Fernandez Astudillo. From softmax to sparsemax: A sparse model of attention and multi-label classification, 2016.

[23] Béatrice Mazoyer, Julia Cagé, Céline Hudelot, and Marie-Luce Viaud. Real-time collection of reliable and representative tweets datasets related to news events.

[24] Kevin Musgrave, Ser-Nam Lim, and Serge Belongie. Pytorch metric learning. https:// github.com/KevinMusgrave/pytorch_metric_learning, 2019.

[25] F. Pedregosa, G. Varoquaux, A. Gramfort, V. Michel, B. Thirion, O. Grisel, M. Blondel, P. Prettenhofer, R. Weiss, V. Dubourg, J. Vanderplas, A. Passos, D. Cournapeau, M. Brucher, M. Perrot, and E. Duchesnay. Scikit-learn: Machine learning in Python. Journal of Machine Learning Research, 12:2825-2830, 2011.

[26] Jeffrey Pennington, Richard Socher, and Christopher D. Manning. Glove: Global vectors for word representation. In Empirical Methods in Natural Language Processing (EMNLP), pages $1532-1543,2014$

[27] Ben Peters, Vlad Niculae, and André F. T. Martins. Sparse sequence-to-sequence models. In Proceedings of the 57th Annual Meeting of the Association for Computational Linguistics, pages 1504-1519, Florence, Italy, July 2019. Association for Computational Linguistics.

[28] Colin Raffel and Daniel P. W. Ellis. Feed-forward networks with attention can solve some long-term memory problems, 2015.

[29] Dyut Kumar Sil, Srinivasan H. Sengamedu, and Chiranjib Bhattacharyya. Readalong: reading articles and comments together. In $W W W, 2011$.

[30] Dyut Kumar Sil, Srinivasan H. Sengamedu, and Chiranjib Bhattacharyya. Supervised matching of comments with news article segments. In CIKM '11, 2011.

[31] Ashish Vaswani, Noam Shazeer, Niki Parmar, Jakob Uszkoreit, Llion Jones, Aidan N. Gomez, Lukasz Kaiser, and Illia Polosukhin. Attention is all you need, 2017.

[32] Xun Wang, Xintong Han, Weilin Huang, Dengke Dong, and Matthew R. Scott. Multi-similarity loss with general pair weighting for deep metric learning, 2019.

[33] Chao-Yuan Wu, R. Manmatha, Alexander J. Smola, and Philipp Krahenbuhl. Sampling matters in deep embedding learning. 2017 IEEE International Conference on Computer Vision (ICCV), Oct 2017.

[34] Sendong Zhao, Yong Huang, Chang Su, Yuantong Li, and Fei Wang. Interactive attention for semantic text matching. arXiv preprint arXiv:1911.06146, 2019. 


\section{Appendices}

\section{A Softmax Expectation}

We will show that an increase in $S$, the length of a document, can be expected to diminish the weight of each token in the softmax step of the attention mechanism. To demonstrate a decrease in $\mathbb{E}\left[\sigma(z)_{i}\right]$ with $S$, we need only show an increase in the expectation on the denominator $\sum_{j=1}^{S} e^{z_{j}}$, which we refer to as expsum.

Using cosine attention, let $\boldsymbol{z}=\cos (\boldsymbol{Q}, \boldsymbol{K})$. Since $\cos (\cdot) \in[-1,1]$, let each $z_{j}$ be an independent random variable drawn uniformly from $\mathcal{U}(-1,1)$. It follows that all $e^{z_{j}} \in\left[e^{-1}, e^{1}\right]$. The expectation on expsum then becomes:

$$
\begin{aligned}
\mathbb{E}\left[\sum_{j=1}^{S} e^{z_{j}}\right] & =\sum_{j=1}^{S} \mathbb{E}\left[e^{z_{j}}\right] \\
& =\sum_{j=1}^{S} \int_{-1}^{1} \frac{1}{2} e^{z_{j}} \\
& =\sum_{j=1}^{S} \frac{e^{2}-1}{2 e} \\
& =S \cdot \frac{e^{2}-1}{2 e} \\
& \approx 1.1752 \cdot S
\end{aligned}
$$

This demonstrates that $\mathbb{E}$ [expsum] increases as a function of $S$. As stated previously, an increase in $\mathbb{E}$ [expsum] decreases $\sigma(\boldsymbol{z})_{i}$. This dictates that $\sigma(\boldsymbol{z})_{i}$ must decrease when $S$ increases. Therefore, an increase in the length of a document will decrease the expected attention weight of each token. 